\title{
Spreading Codes Enables the Blind Estimation of the Hemodynamic Response with Short-Events Sequences
}

\author{
M. A. Lopez-Gordo* \\ ISAER Department, University of Cadiz, c/Chile 1, 11002 Cadiz, Spain \\ TSTC Department, University of Granada, 18071, Granada, Spain \\ Nicolo Association, Churriana de la Vega, Granada, Spain \\ malg@ugr.es \\ D. Sánchez-Morillo \\ ISAER Department, University of Cadiz \\ c/Chile 1, 11002 Cadiz, Spain \\ daniel.morillo@uca.es \\ Marcel A. J. Van Gerven \\ Radboud University Nijmegen Donders Institute for Brain \\ Cognition and Behaviour \\ Nijmegen, Montessorilaan 3, 6525 HR, The Netherlands \\ m.vangerven@donders.ru.nl
}

Accepted 30 October 2014

Published Online 24 December 2014

\begin{abstract}
Finite impulse response (FIR) filters are considered the least constrained option for the blind estimation of the hemodynamic response function (HRF). However, they have a tendency to yield unstable solutions in the case of short-events sequences. There are solutions based on regularization, e.g. smooth FIR (sFIR), but at the cost of a regularization penalty and prior knowledge, thus breaking the blind principle. In this study, we show that spreading codes (scFIR) outperforms FIR and sFIR in short-events sequences, thus enabling the blind and dynamic estimation of the HRF without numerical instabilities and the regularization penalty. The scFIR approach was applied in short-events sequences of simulated and experimental functional magnetic resonance imaging (fMRI) data. In general terms, scFIR performed the best with both simulated and experimental data. While FIR was unable to compute the blind estimation of two simulated target HRFs for the shortest sequences (15 and 31 events) and sFIR yielded shapes barely correlated with the targets, scFIR achieved a normalized correlation coefficient above 0.9 . Furthermore, scFIR was able to estimate in a responsive way dynamic changes of the amplitude of a simulated target HRF more accurately than FIR and sFIR. With experimental fMRI data, the ability of scFIR to estimate the real HRF obtained from a training data set was superior in terms of correlation and mean-square error. The use of short-events sequences for the blind estimation of the HRF could benefit patients in terms of scanning time or intensity of magnetic field in clinical tests. Furthermore, shortevents sequences could be used, for instance, on the online detection of rapid shifts of visual attention that, according to literature, entails rapid changes in the amplitude of the HRF.
\end{abstract}

Keywords: BOLD; fMRI; spreading codes; FIR; hemodynamic response.

\footnotetext{
${ }^{*}$ Corresponding author.
} 


\section{Introduction}

The accurate estimation of the hemodynamic response function (HRF) is a challenging topic justified by clinical inference derived from the morphologic analysis. In the past, many researchers have developed abundant methods for this purpose. Here, we briefly mention some significant works, such as Refs. 1-3. In Ref. 4, the wavelet transform was presented as a rich source of new techniques to enhance analysis of fMRI data. More recently, in Ref. 5 a method based on nonparametric statistics proved to require significantly less computation and the same order of robustness than other methods. Currently, various approaches have been used that typically fit into parametric and nonparametric models. ${ }^{6}$

In parametric models, the HRF is synthetically rebuilt based on the expected similitude to one among a set of basis functions. ${ }^{7-9}$ Models are given the use of specific shapes as templates (e.g. Poisson, Gamma or Gaussian), which are tuned by means of few parameters, namely onset time, time-to-peak, peak amplitude and main to secondary amplitude ratio. This facilitates their estimation but models based on the assumption that HRFs across subjects share the same functional shape for a given region of interest (ROI) and stimulus ${ }^{6}$ will fail in the case of altered blood-oxygen-level dependent (BOLD). Studies have shown that the HRF can be absent, ${ }^{10,11}$ reduced, ${ }^{12,13}$ negative, ${ }^{14,15}$ delayed, ${ }^{16}$ with latencies to peak longer than expected, ${ }^{14,17}$ or with deeper initial dips. ${ }^{16}$ These considerations provide a justification for the use of blind methods for HRF estimation, such as the nonparametric models.

In nonparametric models, there are no assumptions about the HRF shape. Among them, the Finite impulse response (FIR) filters are considered the least constrained option and much more flexible than parametric models. ${ }^{18}$ Unfortunately, estimation of the filter coefficients (beta coefficients) is not exempt from drawbacks. For instance, as the filter order increases, the degrees of freedom decrease with a potential risk of overfitting. Another problem arises when using the standard approach to estimating beta coefficients, as follows:

$$
\beta_{\mathrm{FIR}}=\left(X^{T} X\right)^{-1} X^{T} Y,
$$

where $X$ is the design matrix containing the stimulus sequence and $Y$ is the vector with the observed values (see Ref. 18 for a complete description of $X$ and $Y$ ). In (1), matrix $X^{T} X$ must be inverted. The inversion of this matrix causes numerical instability when the ratio of the number of observations to the filter order is small. ${ }^{18}$ Finally, another problem is that FIR solutions tends to be very noisy without high-quality data as many separate coefficients must be estimated. ${ }^{19}$ The latter necessarily gives rise to long-events sequences for a better performance. In order to mitigate this problem, a solution based on matrix regularization has been proposed. ${ }^{7,18,20}$ The sFIR model (2) consists on the addition in (1) of a smoothing or regularization term that introduces priors about a standard HRF response, thus biasing the beta coefficients, deteriorating the estimation in case of the existence of an altered BOLD and breaking the blind principle. Please, confront (7) for the definition of the variables of the regularization term.

$$
\beta_{\mathrm{SFIR}}=\left(X^{T} X+\sigma^{2} \Sigma^{-1}\right)^{-1} X^{T} Y .
$$

Spreading codes are an alternative to FIR and sFIR in case of short-events sequences. The use of spreading codes in fMRI studies (e.g. m-sequences) is not new. In Ref. 21, m-sequences showed better efficiency than random sequences. In Ref. 22, m-sequences were used to obtain a high-sensitivity estimates of HRF. In Ref. 23, the same author used $\mathrm{m}$-sequences to minimize the impact of nonlinearities on the estimation of the HRF. In Ref. 24, a genetic algorithm was used to find the optimal experimental design for a multi-objective experiment and designs were compared with m-sequences. However, as far as we know, there are no studies that propose spreading codes as an alternative to FIR and sFIR for the blind estimation of the HRF with short-events sequences.

An area of application of scFIR could be the online detection of shifts of visual attention with short-events sequences. It has been proved that visual spatial attention modulates the shape of the HRF in regions of the cortex involved in this cognitive task. For instance, in Ref. 25 changes in the amplitude of the HRF were observed in an eccentricity-dependent manner. Also, in Ref. 26 variations on the latency of the HRF response were studied in contra-lateral and ipsilateral visual cortex. Despite shifts in visual attention is a cognitive ability that humans perform instantaneously, the detection in these studies was performed offline by using 
long-event sequences. Our scFIR approach could enable the online detection by means of short-events sequences in Brain-computer interface $(\mathrm{BCI})^{27-32}$ applications.

In this study, we present and alternative to FIR and sFIR for the blind estimation of the HRF based on short sequences of spreading codes. In this case, FIR and sFIR yield unstable and biased results, respectively. Therefore, scFIR could be an efficient option when the clinical testing requires short scanning time, online analysis of functional capacities or the intensity of the scanner is limited. Another advantage of scFIR is that, while FIR and sFIR require independent and identically normally distributed (i.i.d.) noise, the only restriction of scFIR is the uncorrelatedness of noise and the spreading code sequence. The latter makes unnecessary the use of pre-whitening filters as in FIR and sFIR case.

\section{Materials and Methods}

\subsection{Underlying principles of scFIR}

Let $i[n], o[n]$ and $h[n]$ denote the input, output and impulse response respectively of a Linear Time Invariant (LTI) system. The output of this LTI system, which is contaminated with noise $e[n]$, represents the BOLD signal, the input represents the sequence of spreading codes and the impulse response represents the HRF. Equation (3) shows the relation between these signals.

$$
o[n]=h[n] * i[n]+e[n] .
$$

Let $\hat{h}[n]$ be our estimation of the impulse response $h[n]$ computed by direct circular cross-correlation of $o[n]$ with $i[n]$. That is,

$$
\hat{h}[n]=o[n] \odot i[n] .
$$

Taking the correlation with respect to $i[n]$ in (4)

$$
\hat{h}[n]=o[n] \odot i[n]=h[n] * r_{i i}[n]+e[n] \odot i[n] .
$$

Assuming (i) uncorrelated input and noise and (ii) the auto-correlation function of the input, namely $r_{i i}[n]$, approaches the unit impulse function $\delta[n]$, we have

$$
\hat{h}[n]=h[n] * r_{i i}[n] \approx h[n] .
$$

Notice that, unlike FIR and sFIR models, no matrix inversion is involved. The only algebraic operation to perform is the free-of-instabilities correlation of vectors. Regarding the presence of noise, the only condition to eliminate the presence of noise from (5) to (6), is the uncorrelatedness of the noise $e[n]$ and the sequence of spreading codes $i[n]$ used as input.

\subsection{Simulated fMRI data}

We evaluated scFIR and compared the performance with FIR and sFIR by using simulated fMRI data. We performed the blind estimation of a target HRF, namely $\mathrm{HRF}_{2}$, as well as a dynamic estimation of a changing-in-amplitude target $\mathrm{HRF}$, namely $\mathrm{HRF}_{1}$. The blind estimation was focused on the ability of scFIR to estimate without priors target HRFs. In the dynamic estimation, the goal was to show the ability of scFIR to estimate dynamic changes in the HRF response as a consequence of simulated shifts of visual attention. In both, we used short-events sequences of spreading codes and the results were compared with FIR and sFIR. Equation (7) defines the regularization term included in (2):

$$
\begin{aligned}
\sum_{i j} & =\nu \exp \left(-\frac{g}{2}(i-j)^{2}\right) ; \quad \text { with } \\
g & =\frac{1}{(7 / \mathrm{TR})^{\psi}}, \quad \frac{\sigma^{2}}{\nu}=1,
\end{aligned}
$$

where $\sigma^{2}$ is the noise level, $\nu$ defines the strength of the prior, $\exp ()$ is the operation $e^{()}, g$ is the smoothness factor, $i$ and $j$ are the row and column respectively of the regularization term and TR is the repetition time. In sFIR, the parameters of the regularization term were set up to values considered optimal in previous relevant studies: ${ }^{7,19}$ in this way, $\psi=0.5$ or $\psi=2$ and $\nu$ conveniently equals the noise level.

In order to compare FIR, sFIR and scFIRs, normalized correlation coefficients $(R)$ were calculated in a run with large number of trials. The normalization of $R$ was performed by dividing the covariance of the estimation and the target HRF by the product of their standard deviations. The correlation of random signals is not normally distributed. Therefore, Fisher transformation was applied and then the mean and confidence intervals of the normalized $R$ computed (the confidence intervals are based on the normal distribution of the Fisher Transform). Afterwards, the inverse Fisher transform was applied for the representation in the results section. 
The estimation of the confidence interval was at a significance level of $\alpha=0.05$. Since scFIR was compared with both FIR and sFIR, the Bonferroni correction was taken into account.

Among several families of spreading codes we chose $\mathrm{m}$-sequences because they have the best autocorrelation properties. However, the auto-correlation function of short m-sequences does not approximate a $\delta[n]$ function required in (7), thus causing distortion in the estimation of HRF. In order to mitigate this effect the output of scFIR was low-pass filtered by a second-order Butterworth filter that was executed forward and reverse to cancel phase shifts. Note that the net effect is a fourth-order filter with $6 \mathrm{~dB}$ of loss at the original $3 \mathrm{~dB}$ cutoff frequency. This filtering cannot be considered a consequence of priors about the HRF shape, but rather a direct consequence of the use of realistic m-sequences with nonideal correlation properties. Therefore, this filtering does not invalidate the blind principle. For this reason, the cutoff frequency was arbitrarily set to a third of sampling rate that, in turns, was arbitrarily set to $1 \mathrm{~s}$.

Numerous simulated BOLD signals were generated by using multi-combinations of m-sequences, various levels of signal-to-noise ratio (SNR) and two targets $\mathrm{HRF}_{1}$ and $\mathrm{HRF}_{2}$. Each combination was obtained by the convolution of one of the $\mathrm{m}$ sequences by one of the HRFs plus the addition of white noise, thus giving rise to simulated BOLD signals of different quality.

In order to obtain ground truth in our simulations, two target HRFs were synthesized. The first one $\left(\mathrm{HRF}_{1}\right)$ was generated by means of two Gamma functions

$$
h[n]=A\left(\frac{t^{\alpha_{1}-1} \beta_{1}^{\alpha_{1}} e^{-\beta_{1} t}}{\Gamma\left(\alpha_{1}\right)}-c \frac{t^{\alpha_{2}-1} \beta_{2}^{\alpha_{2}} e^{-\beta_{2} t}}{\Gamma\left(\alpha_{2}\right)}\right)
$$

with standard parameters $\left(\alpha_{1}=6, \alpha_{2}=16, \beta_{1}=1\right.$, $\beta_{2}=1, c=1 / 6, A=1$ ) as proposed in literature ${ }^{7}$ (see Fig. 1, left). We also emulated a real hemodynamic response $\left(\mathrm{HRF}_{2}\right)$ obtained from a patient suffering of aphasia (Fig. 1, Right). In that experiment, a set of words and pseudowords were displayed and participants had to perform a lexical decision to each stimulus (see Ref. 14 for a total description). The idea behind, is the use of a real HRF taken from the

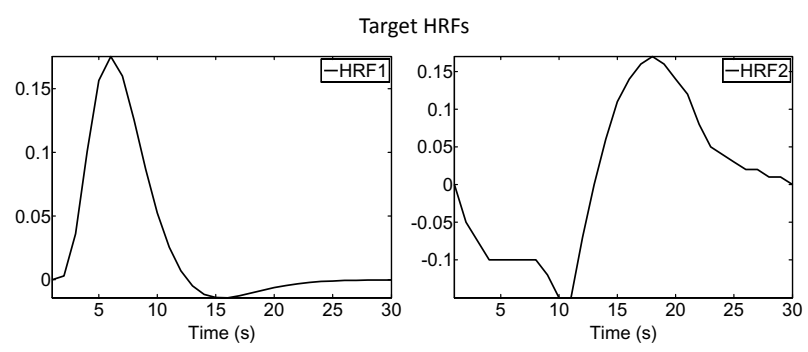

Fig. 1. On the left, target $\mathrm{HRF}_{1}$ is generated by two Gamma functions. On the right, target $\mathrm{HRF}_{2}$ corresponding to the real HRF of a patient suffering of aphasia. ${ }^{14}$ Both target HRFs are composed of 30 beta coefficients. $Y$-axis in arbitrary units.

real world with totally different envelop as the classical $\mathrm{HRF}_{1}$. This would reinforce the blind character of the estimation.

The SNR levels, namely -6 and $6 \mathrm{~dB}$ were chosen as representative of BOLD signals with poor and good quality respectively because typical fMRI signals likely fit within this interval. Furthermore, simulation of BOLD signals with good quality points out the performance justified only by the method without affection of signal with low quality. The sequence lengths (SLs) were chosen as representative of short $(15,31)$, medium (63) and large (127) events sequences. In this study, we are interested in the short-events sequences. Medium and large sequences are shown in order to see the equivalence of the three methods in terms of performance with the increase in the number of events.

\subsubsection{Blind and dynamic estimation}

The blind estimation consisted on the estimation of the target $\mathrm{HRF}_{2}$ (see Fig. 1 right) from various simulated BOLD signals with good and bad quality (SNR $=-6$ and $6 \mathrm{~dB}$, respectively) generated by short-events sequences of 15, 31, 63 and 127 events.

For the dynamic estimation, we simulated sequences of on/off attention to visual stimuli. According to literature, ${ }^{25}$ the amplitude of the HRF can change $60 \%$ and $20 \%$ for eccentricities smaller and greater than $2.5^{\circ}$ and $8.5^{\circ}$, respectively in V1 region between the Attend and the Ignore condition in covert attention paradigms. Since changes in attention can be performed instantaneously by human, dynamic estimation would account for the 


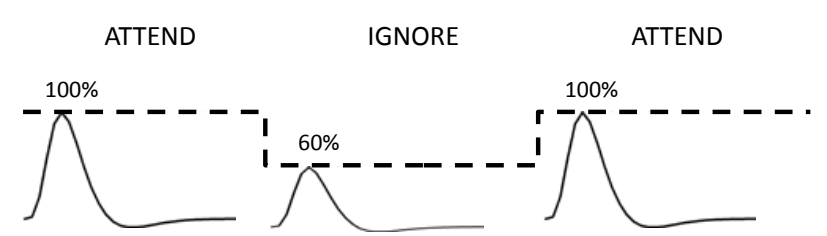

Fig. 2. Shifts in visual attention to stimuli were simulated by means of changes in the amplitude of the HRF. The figure represents a sequence of conditions A-I-A and the corresponding target HRF. The dotted line corresponds to the measurable "level" of attention.

rapid transition between the attend/ignore conditions. Shifts in visual attention to stimuli were simulated by means of changes in the amplitude of the $\mathrm{HRF}$ of $60 \%$ of the maximum value when the stimulus is under the Ignore condition (see Fig. 2). The figure represents a sequence of two conditions Attend-Ignore-Attend and the corresponding HRF. The goal of the three methods was the dynamic and accurate estimation of the amplitude of the target HRF. In this section, we used the target $\mathrm{HRF}_{1}$ to generate the BOLD signals. We considered the 6 th beta coefficient as the estimated amplitude (see amplitude of $\mathrm{HRF}_{1}$ in Fig. 1, left).

In order to generate the "level" of attention with values $100 \%$ and $60 \%$ (see Fig. 2), a continuous estimation of the HRF was performed by feeding the three FIR, sFIR and scFIR methods with a sliding window of simulated BOLD signals (window size $=$ the SL; window shift $=1$ event). The circular correlation properties of the m-sequences, guaranteed the best correlation properties in any of the sliding window. The duration of each condition was approximately 100 times the duration of the SL in event units. Note that we used a sampling rate (or event unit) of $1 \mathrm{~s}$ and 30 beta coefficients. That means that each target HRF lasted $30 \mathrm{~s}$. The latter guaranteed to reach the permanent regime much before a condition transition. Finally, the output of the three methods was smoothed with the same lowpass filtering. Since the "level" of attention remains constant within each $\mathrm{A}-\mathrm{I}-\mathrm{A}$ block (see dotted line in Fig. 2), this filtering caused the elimination of $a$ priori unnecessary high frequency components in the sliding estimation of the HRF amplitude. Therefore, this filtering does not invalidate the blind principle of the estimation. It is only the consequence of prior knowledge of the fMRI block design and not of that of the HRF.

\subsection{Experimental data}

An experimental data set from previous fMRI studies $^{33,34}$ was used to test our method. Please refer to Ref. 33 for a comprehensive explanation about the stimulus characteristics, data acquisition and preprocessing methods. A brief summary follows below.

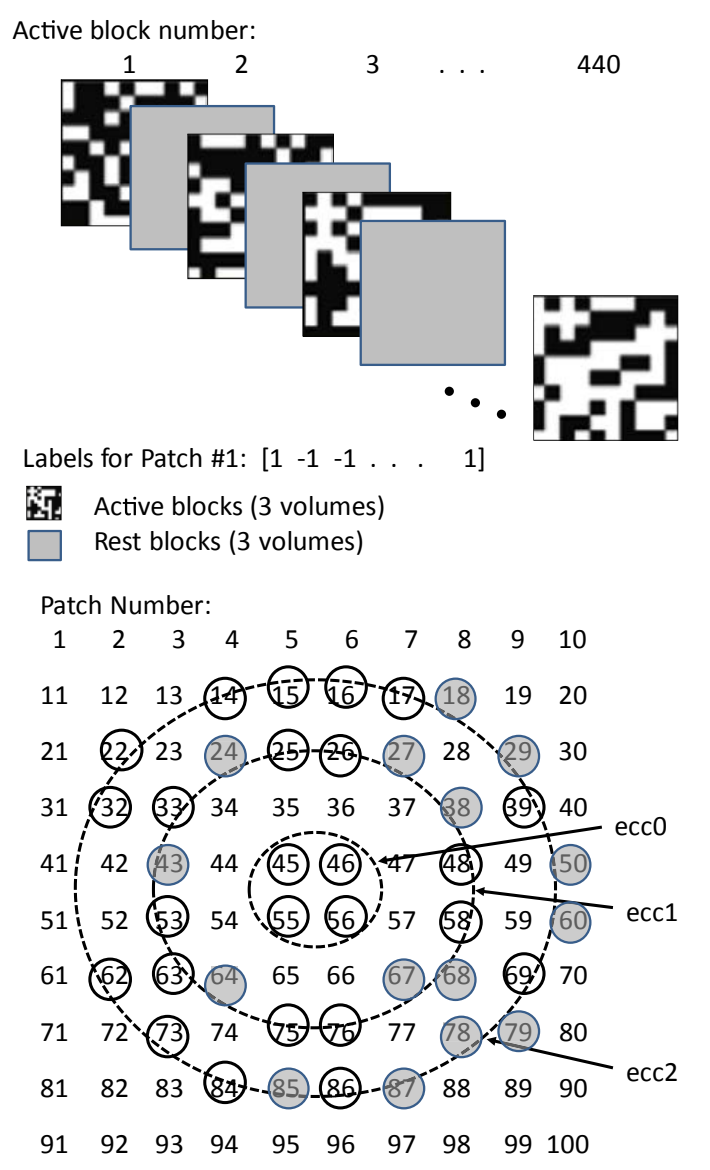

Fig. 3. Upper: Sequence of 440 blocks (and the interleaved rest blocks) consisting of binary-contrast$10 \times 10$ patches. As a sample, the sequence of labels $[1,-1,-1 \ldots 1]$ corresponds to patch number 1 of each image (upper left corner). Adapted from Ref. 33. Bottom: Patch numbering. During the stimuli presentation, subjects under tests gazed at the center of the images. There are three dotted circles demarking iso-eccentricity sets (ecc0, ecc1 and ecc2). Small circles denote candidate patches for the iso-eccentricity sets. Small filled circles denote excluded patches due to an abnormal activation of the associated voxel. 
MRI data were obtained with a 3.0-Tesla Siemens MAGNETOM Trio A Tim scanner located at the ATR Brain Activity Imaging Center (Japan). The acquired fMRI data were slice-timing and motion corrected using SPM2. Basic pre-processing such as linear trend removal and normalization was applied. The main objective of Ref. 33 was the reconstruction of binary-contrast $10 \times 10$-patch images from voxel activity. For this purpose, a random-block design in an fMRI experiment was built with 20 runs, 22 different blocks per run and one random image per block, thus giving rise to 440 different random images (see Fig. 3 left) and three volumes per block. Each block (both active and inactive) lasted $6 \mathrm{~s}$ and contained three volumes $(\mathrm{TR}=2 \mathrm{~s})$. In each block, an image consisting of $10 \times 10$ random binary-contrast patches was shown. Each of the 100 patches was either flickering at $6 \mathrm{~Hz}$ ("1" or active condition) or a homogeneous gray ("0" or inactive condition) with equal probability.

The objective of this section is the estimation of the experimental target HRFs from various voxels arranged in iso-eccentricity sets. The retinotopy principle links specific visual field locations to specific cortical location, thus forming a mapping from the visual field to the cortical voxels. ${ }^{35,36}$ Due to the magnification factor, ${ }^{37,38}$ this spatial mapping is oneto-many for central patches and many-to-one at the periphery (i.e. the activity of voxels mapped to central and periphery patches is likely caused just by one and many stimulus, respectively). In other words, the visual receptive field of receptors in the central area is smaller than that of the periphery. That means that stimuli from central patches are expected to cause activations in more voxels than those coming from the periphery.

Conversely, voxels that according to retinotopy respond to stimuli from the periphery will cause fMRI signals associated to more than one patch, so these voxels are contaminated with noise caused by neighboring stimuli. The immediate consequence is that BOLD signals extracted from voxels activated by periphery will have components correlated with more stimuli than those activated by central vision. Based on this knowledge we would expect voxels activated by patches situated at a certain eccentricity to have a HRF with similar amplitude. For this reason we chose three sets of patches arranged by crescent eccentricity, namely ecc0, ecc1 and ecc2. In each set, the patch number closer to the iso-eccentricity circle was included (see Fig. 3, right). For each patch of each iso-eccentricity set, the voxel with the higher SNR was found and associated to the patch. The SNR of each voxel related to a patch, was computed as the energy of the sequence label of that patch divided by the variance of the BOLD signal once the sequence label was removed. The BOLD signal of each voxel was composed of 440 blocks. It was divided in training data (380 blocks or 2280 events) and evaluation data (60 blocks or 360 events). For each voxel, we estimated the HRF with the training data. It was considered the target HRF. The target HRF was estimated as the mean value of SFIR and scFIR estimations (FIR was not taken into account due to numerical instability). Then, we estimated the target HRF from the evaluation data. Since voxels from each iso-eccentricity set are located in different brain areas (upper-lower and left-right hemispheres), each of the estimated HRF could not share the same shape. For this reason, we discarded voxels within each iso-eccentricity set whose HRF was clearly different from the others. It corresponds to voxels associated to patches filled in gray in Fig. 3, right. Afterwards, the number of remaining voxels in each iso-eccentricity set was four, nine and twelve for ecc0, ecc1 and ecc2 respectively (see patches within unfilled circles in Fig. 3, right). The evaluation data were submitted to FIR, sFIR and scFIR methods to estimate the HRF with small number of samples. Normalized $R$ and mean-square error was computed. As with the simulated data, Fisher transform was used before mean values of $R$ were obtained. Also, Bonferroni correction was applied $(\alpha=0.05)$.

\subsection{Structured versus white noise}

In the simulated section (blind and dynamic estimation) white noise was added to the BOLD signal. We did it since FIR and sFIR require white noise for an optimum performance, thus favoring them in the comparison with scFIR. There are multiple sources of low-frequency structured noise in fMRI signals (e.g. hardware imperfections, heart rate, and respiration, movement artifacts, etc.). These contributions give rise to a certain level of autocorrelation in the residuals of the fMRI signal, thus invalidating the statistical analysis. ${ }^{39}$ In the presence of structured noise, some pre-whitening filters (or high pass 
filters) are typically used for optimal performance of FIR and sFIR. However, this restriction does not apply for scFIR, whose performance only relies on the uncorrelatedness of noise and the spreading codes (see Eqs. (5) and (6)). This is a much more flexible restriction that permits the typical presence of structured noise in fMRI signals without the need of pre-whitening filters.

\section{Results}

\subsection{Blind estimation}

This section shows beta coefficients estimated by FIR, sFIR and scFIR with simulated BOLD signals. BOLD signals were simulated by several combinations of SNRs $(\mathrm{SNR}=-6$ and $6 \mathrm{~dB}), \mathrm{SLs}(\mathrm{SL}=15$,

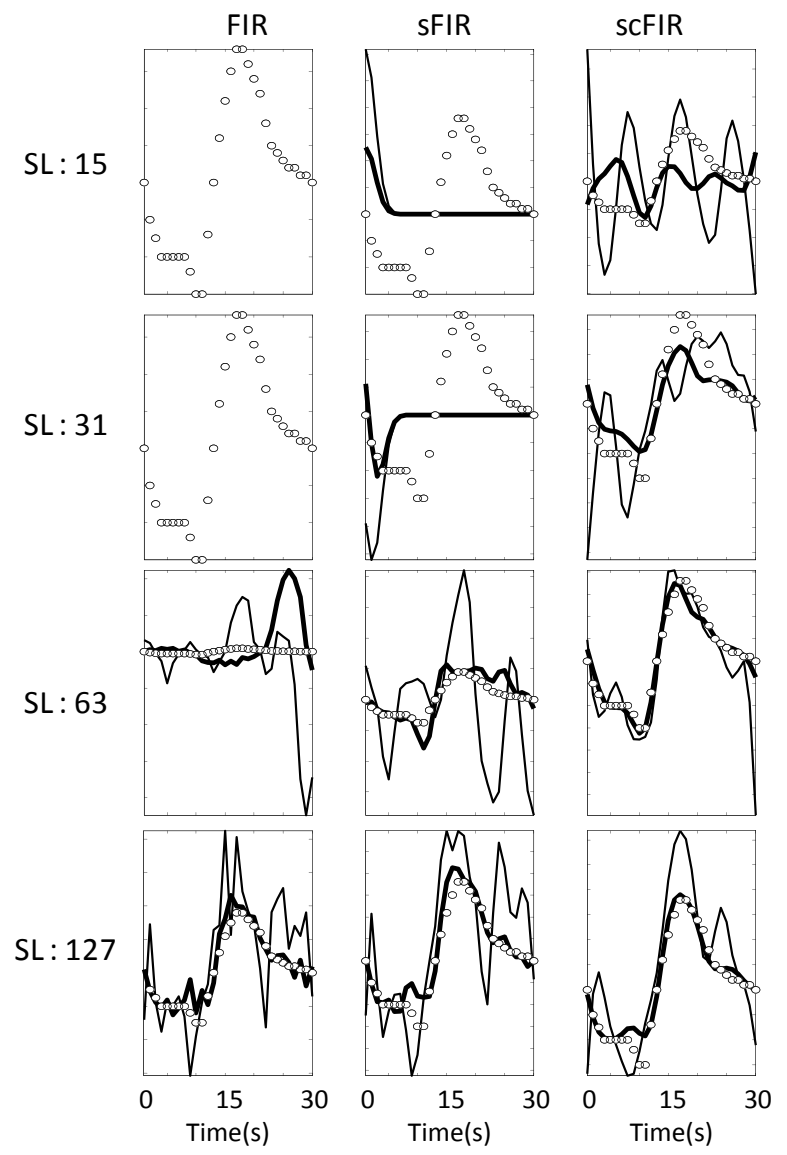

Fig. 4. Beta coefficients estimated by FIR, sFIR and scFIR. In each plot, the circles represent the target $\mathrm{HRF}_{2}$ (see Fig. 1, right), the thin and thick lines represent the estimated beta coefficient with bad and good BOLD signal quality (SNR $=-6$ and $6 \mathrm{~dB}$, respectively). SL: sequence length in events units.
31, 63 and 127) and the target $\mathrm{HRF}_{2}$. Plots without curves (e.g. in FIR column, SL $=15$ and 31) means inability to estimate coefficients due to numerical instability. Figure 4 shows a representative example of an estimation of the target $\mathrm{HRF}_{2}$ (small circles).

For statistical purposes, we estimated the target $\mathrm{HRF}_{2} 1000$ times for each combination of BOLD signals (except for $\mathrm{SL}=127$, for which we performed 4000 estimations). Figure 5 shows the mean normalized $R$ and confidence intervals (see Sec. 2.2 for further details about their computation). The high number of estimations guaranteed hardly visible confidence intervals, thus facilitating the search for significant differences in performance by visual inspection. Notice that for $\mathrm{SL}=15$ and 31, FIR bars are missing due to numerical instability.

\subsection{Dynamic estimation}

This section shows the estimation of the "level" of attention (dotted line in Figs. 2 and 6) by FIR, sFIR and scFIR. BOLD signals were simulated by multiple combinations of SNRs (SNR $=-6$ and $6 \mathrm{~dB}$ ),
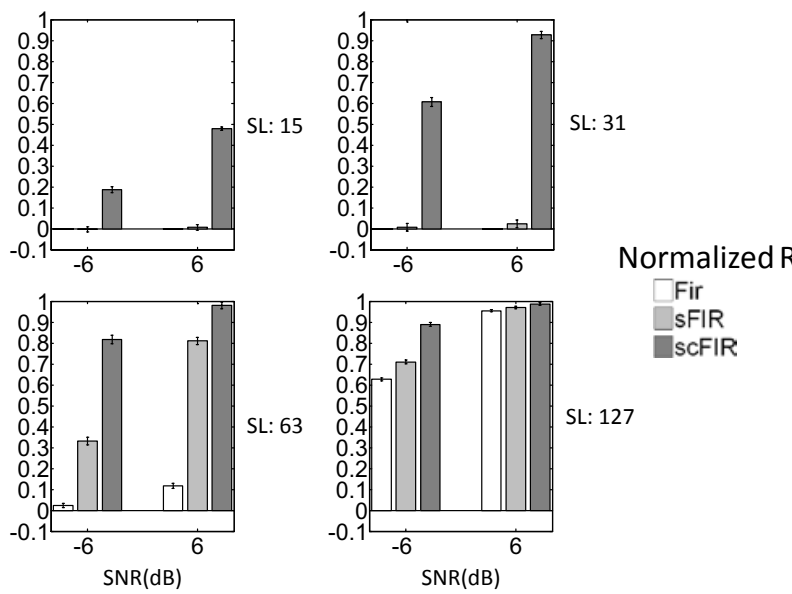

Fig. 5. Normalized $R$ and confidence intervals for the estimation of target $\mathrm{HRF}_{2}$ by FIR, sFIR and scFIR. Several simulated BOLD signals were generated as a combination of different SLs (SL = 15, 31, 63 and 127), different signal qualities $(\mathrm{SNR}=-6$ and $6 \mathrm{~dB}$ ) and two target $\mathrm{HRF}$ s $\left(\mathrm{HRF}_{1}\right.$ and $\left.\mathrm{HRF}_{2}\right)$. For $\mathrm{SL}=15$ and 16 , FIR was unable to compute the beta coefficients and sFIR achieved a nearly uncorrelated estimation of the target $\mathrm{HRF}_{2}$. For all values of SL, the normalized $R$ of scFIR significantly outperformed that of FIR and sFIR. SL: sequence length in events units. 
SLs (SL $=15,31,63$ and 127) and target $\mathrm{HRF}_{1}$. The "level" of attention was considered the 6 th estimated beta coefficient, which corresponds to the amplitude of target $\mathrm{HRF}_{1}$ (see Fig 1. left). We simulated shift of visual attention by means of modulation of this amplitude between values $100 \%$ and $60 \%$ (Attend and ignore conditions, respectively). Plots without curves (e.g. in FIR column, SL $=15$ and 31) means inability to estimate coefficients due to numerical instability. Figure 6 shows a representative example of estimation of target $\mathrm{HRF}_{1}$.

For statistical purposes, we estimated the "level" of attention by estimating the amplitude of the HRF more than 6000 times per condition (sequence A-I-A

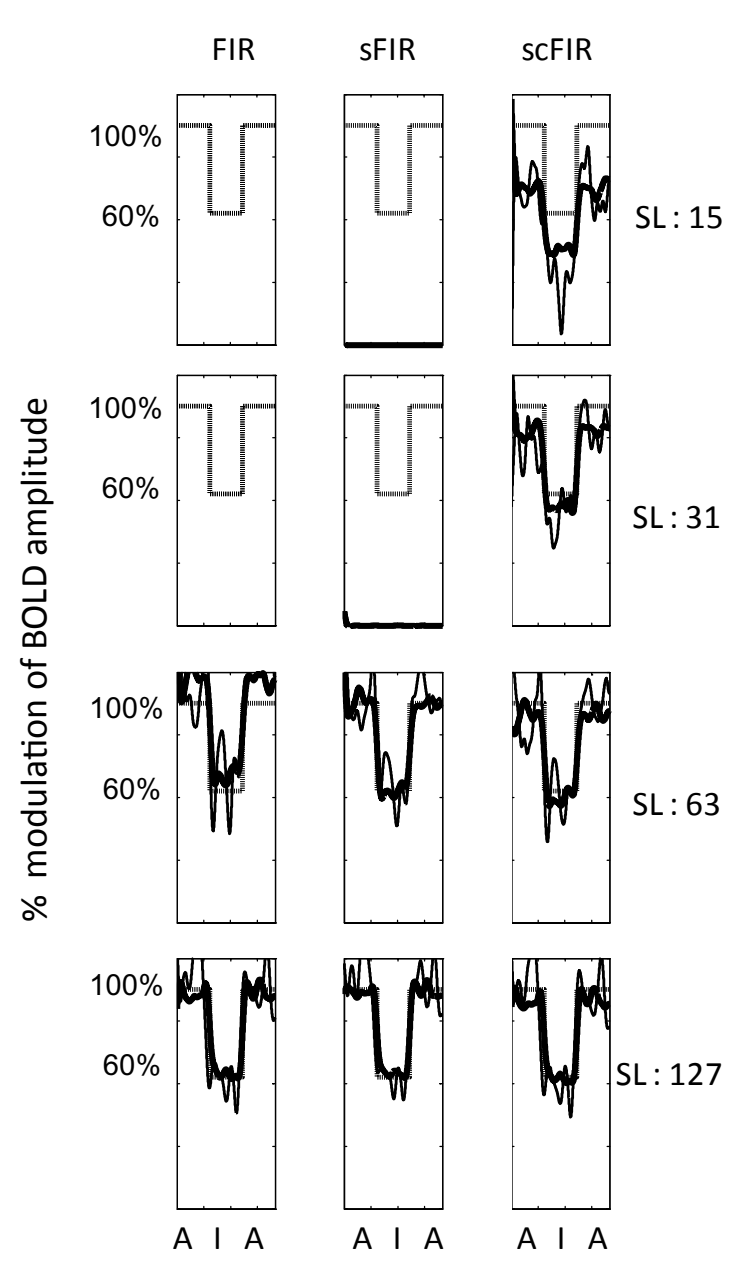

Fig. 6. Estimation of the "level" of attention (dotted line) by FIR, sFIR and scFIR during the Attend (A) and Ignore (I) conditions. The thin and thick lines represent the estimated amplitude with bad and good BOLD signal quality $(\mathrm{SNR}=-6$ and $6 \mathrm{~dB}$, respectively. SL: sequence length in sequence units.
Table 1. Normalized $R$ and confidence intervals ( $\alpha=$ $0.05)$ for the estimation of the "level" of attention. Cells with gray backgrounds mean values of $R$ significantly better than the other two methods. Note that FIR was unable to compute the beta coefficient due to numerical instability for SL values up to 31, while scFIR estimated them with an extraordinary high correlation $(R=0.96)$.

\begin{tabular}{|c|c|c|c|c|c|}
\hline SL & $\operatorname{SNR}(d B)$ & $x$ FIR & $R \min$ & $R$ & $R \max$ \\
\hline \multirow[t]{6}{*}{15} & \multirow[t]{3}{*}{-6} & FIR & $\mathrm{NaN}$ & $\mathrm{NaN}$ & $\mathrm{NaN}$ \\
\hline & & sFIR & -0.65 & -0.64 & -0.63 \\
\hline & & scFIR & 0.79 & 0.80 & 0.80 \\
\hline & \multirow[t]{3}{*}{6} & FIR & $\mathrm{NaN}$ & $\mathrm{NaN}$ & $\mathrm{NaN}$ \\
\hline & & sFIR & -0.66 & -0.65 & -0.65 \\
\hline & & scFIR & 0.93 & 0.93 & 0.93 \\
\hline \multirow[t]{6}{*}{31} & \multirow[t]{3}{*}{-6} & FIR & $\mathrm{NaN}$ & $\mathrm{NaN}$ & $\mathrm{NaN}$ \\
\hline & & sFIR & -0.01 & 0.01 & 0.02 \\
\hline & & scFIR & 0.85 & 0.85 & 0.86 \\
\hline & \multirow[t]{3}{*}{6} & FIR & $\mathrm{NaN}$ & $\mathrm{NaN}$ & $\mathrm{NaN}$ \\
\hline & & sFIR & 0.04 & 0.06 & 0.07 \\
\hline & & scFIR & 0.96 & 0.96 & 0.96 \\
\hline \multirow[t]{6}{*}{63} & \multirow[t]{3}{*}{-6} & FIR & 0.54 & 0.55 & 0.56 \\
\hline & & sFIR & 0.82 & 0.83 & 0.83 \\
\hline & & scFIR & 0.84 & 0.85 & 0.85 \\
\hline & \multirow[t]{3}{*}{6} & FIR & 0.94 & 0.94 & 0.94 \\
\hline & & sFIR & 0.96 & 0.97 & 0.97 \\
\hline & & scFIR & 0.96 & 0.96 & 0.96 \\
\hline \multirow[t]{6}{*}{127} & \multirow[t]{3}{*}{-6} & FIR & 0.83 & 0.83 & 0.84 \\
\hline & & sFIR & 0.89 & 0.89 & 0.89 \\
\hline & & scFIR & 0.82 & 0.82 & 0.83 \\
\hline & \multirow[t]{3}{*}{6} & FIR & 0.97 & 0.97 & 0.97 \\
\hline & & sFIR & 0.97 & 0.97 & 0.98 \\
\hline & & scFIR & 0.97 & 0.97 & 0.97 \\
\hline
\end{tabular}

in Figs. 2 and 6). Table 1 shows the normalized $R$ and confidence intervals between the simulated "level" of attention and the estimated one. Gray backgrounds represent significant differences. Notice that for $\mathrm{SL}=15$ and 31 , FIR was unable to compute the HRF due to numerical instability.

\subsection{Experimental fMRI data}

Figure 7 shows statistical results from experimental fMRI data. FIR results were very poor in comparison with sFIR and scFIR and were not plotted. In this case, the use of sFIR is the simplest workaround to the numerical instability of FIR. 


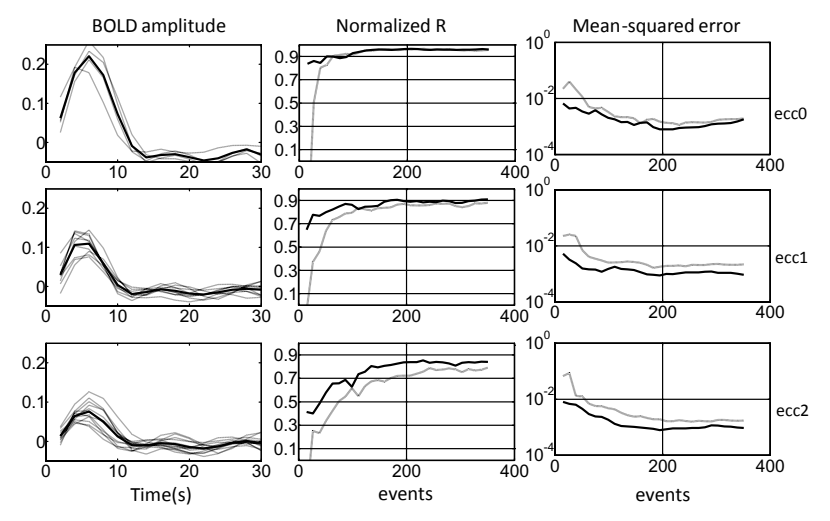

Fig. 7. Estimation of target HRF by sFIR and scFIR from experimental BOLD signals. First column: dotted and thick lines represent the estimated target $\mathrm{HRF}$ and the average target $\mathrm{HRF}$ in each iso-eccentricity set. The average target HRF is plotted only to visualize the homogeneity of the estimated target HRF within each eccentricity set. $X$-axis in seconds. $Y$-axis corresponds to the BOLD amplitude. Second column: the dotted and thick lines represent the mean normalized $R$ between the target HRF (estimated from the training data) of each voxel and the estimated HRF (estimated from the evaluation data) for sFIR and scFIR, respectively. $X$-axis in events units. Third column: the dotted and thick lines represent the mean-square error between the target HRF and the estimated HRF for sFIR and scFIR, respectively. $Y$-axis in logarithms units. $X$-axis in events units.

\section{Discussion}

\subsection{Blind estimation}

Figure 4 shows single-trial estimations of the target $\mathrm{HRF}_{2}$ for different values of SNR and SL. In this way, the shape of each estimation can be visually inspected without benefitting from any denoising process caused by trials averaging. Notice that for the shortest SLs (SL $=15$ and 31), FIR was unable to compute the HRF and sFIR yielded rather abnormal shape. The FIR result is justified because the ratio of the number of independent data to the filter order is small (the filter order was 30 and the fMRI data were 15 and 31). In this case, the matrix tends to be badly conditioned and the solution becomes unstable in the case of FIR. ${ }^{18}$ The abnormal shape estimated by sFIR could be justified due to a suboptimal adjusting of the parameter $\psi$ in (7). In Sec. 2.2, we described the two values considered optimal in two studies. Despite this blind estimation without priors about the target HFR, we favored sFIR method by trying both values and presenting the best result in
Fig. 4. Finally, it can be observed that scFIR is the only approach that, with small number of observations and good SNR $(\mathrm{SL}=31, \mathrm{SNR}=6 \mathrm{~dB})$ was able to perform a reasonable estimation of the beta coefficients without instability but with a high correlation (see Fig. 5, upper right plot). However, we must keep in mind that Fig. 4 only reflects single-trial estimations of target $\mathrm{HRF}_{2}$, thus without validity from a statistical point of view.

Figure 5 shows the mean of the normalized $R$ between the target $\mathrm{HRF}_{2}$ and their estimations. It also shows the confident intervals. The number of trials used to compute this statistics was as high as necessary to reduce the confident intervals to a bearable-visible level (see Sec. 3.1). In this way, significant differences in normalized $R$ between methods can be pointed out just by visual inspection. For the shortest SLs (SL $=15$ and 31), FIR was unable to compute the HRF, sFIR approximately yielded an uncorrelated shape and scFIR reported an extraordinary normalized $R$, that, in the case of BOLD signal with good quality $(\mathrm{SNR}=6 \mathrm{~dB}$, $\mathrm{SL}=31$ ) exceeded 0.9 (see Fig. 5, upper right plot). For the medium and long SLs (SL $=63$ and 127), the difference in performance of FIR, sFIR and scFIR gradually disappear. In the best scenario $(\mathrm{SL}=127$, $\mathrm{SNR}=6 \mathrm{~dB}$ ), the difference in performance between the three methods is very small, and despite it is significantly different in favor of scFIR, it suggests that longer SLs would cause total convergence in performance.

In summary, and despite we favored sFIR by adjusting the $\psi$ parameter, we state that in terms of normalized $R$, scFIR performed significantly better than FIR and sFIR with short-events sequences and simulated BOLD signals.

\subsection{Dynamic estimation}

We can state similar appreciations as in the discussion of the blind estimation. In summary, for short sequences ( $\mathrm{SL}=15$ and 31$), \mathrm{FIR}$ and sFIR were unable to compute the amplitude of the HRF with accuracy (see Fig. 6, first and second columns, first and second rows), whereas scFIR was able to compute the amplitude with accuracy (e.g. normalized $R$ between 0.80 and 0.96 , see Table 1 ). The estimation of the HRF has a delay of exactly the SL (e.g. for $\mathrm{SL}=127$, the three methods would output 
the estimated HRF with a delay of 127 events). This is a key fact for the online detection of modulations of amplitude (e.g. in paradigms related to shifts of visual attention). For instance, in Table 1, scFIR matches the "level" of attention (dotted line in Fig. 6) with a normalized $R$ between 0.80 and 0.96 for $\mathrm{SL}=15$ and 31 , respectively). In the case of a $\mathrm{TR}=250 \mathrm{~ms}$, shifts of visual attention causing a $60 \%$ decrement of the amplitude of the HRF could be detected within 3.8 and $7.9 \mathrm{~s}$ respectively.

Table 1 shows that for the shortest SLs (SL $=15$ ad 31) and with simulated BOLD signals, scFIR performed the detection of shifts of attention significantly better than FIR and sFIR.

\subsection{Experimental fMRI data}

Figure 7 shows the experimental target HRF (first column) and the mean normalized $R$ and meansquare error (second and third column) of isoeccentricity sets ecc0, ecc1 and ecc 2 . The FIR performance was far worse from that obtained by sFIR and scFIR. For this reason FIR curves were not plotted.

Each plot in first column shows all targets HRFs corresponding to each one of the voxels that belongs to a specific voxel set (dotted thin lines). The thick line corresponds to the averaged HRF in the isoeccentricity set. The latter is only plotted as a visual reference that was used to discard voxels with an unusual or deviant HRF. It can be observed in Fig. 7 an interesting decrement of the amplitude of the target HRF with the increment of the eccentricity (approximately $0.22,0.11$ and 0.08 for ecc0, ecc1 and ecc2, respectively). The latter is consistent with the fundaments explained in Sec. 2.3. In the second and third column, scFIR obtains always a better normalized $R$ and mean-square error than sFIR for the shortest number of events that gradually converges with the increasing events. It can be observed that the number of events needed for the convergence in performance between sFIR and scFIR decrease with the eccentricity (i.e. the number of events needed in ecc0 is smaller than in ecc1, tat in turns, smaller than ecc2). We justify this in the better quality of BOLD signal of voxels with smaller eccentricity respect to the stimulus label. This is consistent with the results showed in Fig. 5 and Table 1, that is, the performance of the three methods approaches themselves with the enhancement of the conditions (SNR and SL).

\subsection{White versus structured noise}

In scFIR, the uncorrelatedness of noise and the input sequence is assumed. This represents an extraordinary advantage of scFIR in comparison with FIR and sFIR. These two methods require i.i.d. noise for an optimal performance, ${ }^{39}$ in broad words, they need white noise. In practice, this leads to the use of pre-whitening filters (typically high-pass filters) that, somehow, means the enhancing of the level of noise. With the scFIR approach this is not necessary because uncorrelatedness is less restrictive than independency. As an example of the latter, we could state that scFIR performs optimal with any fMRI signal contaminated by structured (nonwhite) noise as long as the noise and the input sequence are uncorrelated to each other. In practical terms, the latter can always be assumed. In this case, both FIR and sFIR will perform suboptimal since structured noise is not i.i.d. noise. For this reason the fMRI signal typically are high-passed to equalize low and high spectral bands of the noise, thus adopting the shape of white noise. This operation is not needed for scFIR. Furthermore, this operation is completely irrelevant for scFIR as long as the uncorrelatedness of noise and the input sequence remains unaltered and, a priori, there is no reason to think so.

The results showed in Secs. 3.1 and 3.2 were obtained with simulated BOLD signals contaminated by white noise, thus giving rise to levels $\mathrm{SNR}=-6$ and $6 \mathrm{~dB}$. As explained before, the use of white noise is necessary for the optimal performance of FIR and sFIR. This is not the case for scFIR, which perfectly tolerates the presence of structured (nonwhite) noise providing that it is uncorrelated with the input sequence. Even in this optimal scenery for FIR and sFIR, scFIR demonstrates much better performance with short sequences.

Some preliminary tests were executed with structured noise (that is noise with a certain level of autocorrelation), obtained by simply low-pass filtering of white noise and the results were, as expected, even better than those presented here with white noise.

\section{Conclusion}

In summary, in the present study we have presented and alternative to FIR and SFIR for the blind and dynamic estimation of the HRF with short-events sequences based on spreading codes. Whereas FIR 
and sFIR yielded unstable solutions and biased solutions, respectively, scFIR presented significant difference in normalized $R$ and mean-square error with short-events sequences.

The scFIR approach can be considered a blind and improved version of FIR and sFIR that performs better with small number of observations, reduces computational complexity, and lacks ill-posedness without the use of a regularization term. It is achieved because in scFIR the estimation of the beta coefficients relies on simple correlation of vectors.

The scFIR approach can be applied in sceneries such as for the blind estimation of BOLD (e.g. in cerebral-vascular impaired patients), when the clinical test requires short scanning time or the intensity of the scanner is limited, and for the online detection of changes in the amplitude of the HRF.

A specific area of application of scFIR could be the online detection of shifts of visual attention, or more generally, the online detection of attention with BCIs. In the case of visual attention, BCIs are designed to detect endogenous attention (covert attention) without muscular movement, thus providing additional information to that provided by other devices such as eye-trackers based on shifts of gaze. In some cases, when a user is unable to shift the gaze correctly (e.g. users with locked-in syndrome or Amyotrophic lateral sclerosis), covert attention is the only way to detect the user visual attention. However, EEG-based BCIs have proved extremely bad performance in detecting visual covert attention. For instance, from the conclusion section of Ref. 40, we can infer that the BCI could only detect visual covert attention at a rate of on one per minute approximately. In this regard and given the delays discussed in Sec. 4.2 and performance of Table 1, we could roughly expect a HRF-based BCI to take less than $10 \mathrm{~s}$ in the detection of visual covert attention, thus constituting a novel and promising paradigm.

\section{Acknowledgments}

This work was supported by Nicolo Association for the $\mathrm{R}+\mathrm{D}+\mathrm{i}$ in Neurotechnologies for disability, the research project P11-TIC-7983, Junta of Andalucia (Spain) and the Spanish National Grant TIN201232030, co-financed by the European Regional Development Fund (ERDF). We thank collaboration of
Tom Heskes, the supervisor of the Institute for Computing and Information Sciences (ICIS), Radboud University, Nijmegen, The Netherlands.

\section{References}

1. E. Zarahn, A trial-based experimental design for fMRI, NeuroImage 6 (1997) 122-138.

2. G. K. Aguirre, E. Zarahn and M. D'Esposito, The variability of human, BOLD hemodynamic responses, NeuroImage 8 (1998) 360-369.

3. K. J. Worsley et al., A general statistical analysis for fMRI data, NeuroImage 15 (2002) 1-15.

4. E. Bullmore et al., Wavelets and functional magnetic resonance imaging of the human brain, NeuroImage 23 (2004) S234-S249.

5. P. A. De Mazière and M. M. Van Hulle, fMRI bold signal analysis using a novel nonparametric statistical method, J. Magn. Reson. 185 (2007) 138-151.

6. T. Zhang, F. Li, L. Beckes and J. A. Coan, A semiparametric model of the hemodynamic response for multi-subject fMRI data, NeuroImage 75 (2013) 136-145.

7. M. A. Lindquist and T. D. Wager, Validity and power in hemodynamic response modeling: A comparison study and a new approach, Hum. Brain Mapp. 28 (2007) 764-784.

8. K. J. Friston, P. Jezzard and R. Turner, Analysis of functional MRI time-series, Hum. Brain Mapp. 1 (1994) 153-171.

9. J. C. Rajapakse, F. Kruggel, J. M. Maisog and D. Y. von. Cramon, Modeling hemodynamic response for analysis of functional MRI time-series, Hum. Brain Mapp. 6 (1998) 283-300.

10. P. M. Rossini, Does cerebrovascular disease affect the coupling between neuronal activity and local haemodynamics? Brain 127 (2004) 99-110.

11. F. Binkofski and R. J. Seitz, Modulation of the BOLD-response in early recovery from sensorimotor stroke, Neurology 63 (2004) 1223-1229.

12. M. Schaaf et al., Functional MR imaging in patients with carotid artery stenosis before and after revascularization, Am. J. Neuroradiol. 31 (2010) 1791-1798.

13. J. Fridriksson, C. Rorden, P. Morgan, K. Leigh Morrow and G. Baylis, Measuring the hemodynamic response in chronic hypoperfusion, Neurocase $\mathbf{1 2}$ (2006) 146-150.

14. B. Bonakdarpour, T. B. Parrish and C. K. Thompson, Hemodynamic response function in patients with stroke-induced aphasia: Implications for fMRI data analysis, NeuroImage 36 (2007) 322-331.

15. J. Röther et al., Negative dip in BOLD fMRI is caused by blood flow- Oxygen consumption uncoupling in humans, NeuroImage 15 (2002) 98-102.

16. A. C. Roc, Altered hemodynamics and regional cerebral blood flow in patients with hemodynamically significant stenoses, Stroke 37 (2006) 382-387. 
17. K. K. Peck, Functional magnetic resonance imaging before and after aphasia therapy: Shifts in hemodynamic time to peak during an overt language task, Stroke 35 (2004) 554-559.

18. C. Goutte, F. A. Nielsen and K. H. Hansen, Modeling the hemodynamic response in fMRI using smooth FIR filters, IEEE Trans. Med. Imaging 19 (2000) 1188-1201.

19. M. A. Lindquist, J. Meng Loh, L. Y. Atlas and T. D. Wager, Modeling the hemodynamic response function in fMRI: Efficiency, bias and mis-modeling, NeuroImage 45 (2009) S187-S198.

20. J. M. Ollinger, M. Corbetta and G. L. Shulman, Separating processes within a trial in event-related functional MRI, NeuroImage 13 (2001) 218-229.

21. G. T. Buračas and G. M. Boynton, Efficient design of event-related fMRI experiments using M-sequences, NeuroImage 16 (2002) 801-813.

22. J. A. De Zwart et al., Temporal dynamics of the BOLD fMRI impulse response, NeuroImage $\mathbf{2 4}$ (2005) 667-677.

23. J. A. De Zwart et al., Hemodynamic nonlinearities affect BOLD fMRI response timing and amplitude, NeuroImage 47 (2009) 1649-1658.

24. M.-H. Kao, A. Mandal, N. Lazar and J. Stufken, Multi-objective optimal experimental designs for event-related fMRI studies, NeuroImage 44 (2009) 849-856.

25. D. W. Bressler, F. C. Fortenbaugh, L. C. Robertson and M. A. Silver, Visual spatial attention enhances the amplitude of positive and negative fMRI responses to visual stimulation in an eccentricitydependent manner, Vis. Res. 85 (2013) 104112.

26. T. Kellermann et al., Latencies in BOLD response during visual attention processes, Brain Res. 1386 (2011) 127-138.

27. J. Li et al., Design of assistive wheelchair system directly steered by human thoughts, Int. J. Neural Syst. 23 (2013) 1350013.

28. N. V. Manyakov, I. Aizenberg, N. Chumerin and M. M. Van Hulle, in Complex-Valued Neural Networks, Chapter 8, ed. A. Hirose (John Wiley \& Sons, Inc., 2013), pp. 185-208.
29. G. R. Müller-Putz, C. Pokorny, D. S. Klobassa and P. Horki, A single-switch bci based on passive and imagined movements: Toward restoring communication in minimally conscious patients, Int. J. Neural Syst. 23 (2013) 1250037.

30. G. Rodríguez-Bermúdez, P. J. García-Laencina and J. Roca-Dorda, Efficient automatic selection and combination of eeg features in least squares classifiers for motor imagery brain-computer interfaces, Int. J. Neural Syst. 23 (2013) 1350015.

31. M. A. Lopez-Gordo, F. Pelayo, A. Prieto and E. Fernandez, An auditory brain-computer interface with accuracy prediction, Int. J. Neural Syst. 22 (2012) $1-14$.

32. M. A. Lopez-Gordo and F. Pelayo, A Binary phaseshift keying receiver for the detection of attention to human speech, Int. J. Neural Syst. (2013), doi:10.1142/S0129065713500160.

33. Y. Miyawaki et al., Visual image reconstruction from human brain activity using a combination of multiscale local image decoders, Neuron 60 (2008) 915929.

34. Y. Miyawaki et al., Visual image reconstruction from human brain activity: A modular decoding approach, J. Phys. Conf. Ser. 197 (2009) 012021.

35. S. A. Engel et al., fMRI of human visual cortex, Nature 369 (1994) 525.

36. M. Sereno et al., Borders of multiple visual areas in humans revealed by functional magnetic resonance imaging, Science 268 (1995) 889-893.

37. P. M. Daniel and D. Whitteridge, The representation of the visual field on the cerebral cortex in monkeys, J. Physiol. 159 (1961) 203-221.

38. E. L. Schwartz, A quantitative model of the functional architecture of human striate cortex with application to visual illusion and cortical texture analysis, Biol. Cybern. 37 (1980) 63-76.

39. T. E. Lund, K. H. Madsen, K. Sidaros, W.-L. Luo and T. E. Nichols, Non-white noise in fMRI: Does modelling have an impact? NeuroImage 29 (2006) 54-66.

40. B. Z. Allison et al., Towards an independent braincomputer interface using steady state visual evoked potentials, Clin. Neurophysiol. 119 (2008) 399-408. 